\title{
Volume change pattern of decompression of mandibular odontogenic keratocyst
}

\author{
Jin Hoo Park ${ }^{1 \dagger}$, Eun-Jung Kwak ${ }^{1+}$, Ki Sung You ${ }^{3}$, Young-Soo Jung ${ }^{1,2}$ and Hwi-Dong Jung ${ }^{1,2^{*}}$ (D)
}

\begin{abstract}
Objectives: This study was aimed to analyze the reducing pattern of decompression on mandibular odontogenic keratocyst and to determine the proper time for secondary enucleation.

Materials and methods: Seventeen patients with OKC of the mandible were treated by decompression. Forty-five series of $\mathrm{CT}$ data were taken during decompression and measured by using InVivo software (Anatomage, San Jose, Calif) and were analyzed.

Results: The expected relative volume during decompression is calculated using the following formula: $V(t)=$ $V_{\text {initial }} \times \exp .\left(a t+1 / 2 b t^{2}\right)(t=$ duration after decompression $($ day $))$. There was no significant directional indicator in the rate of reduction between buccolingual and mesiodistal widths.

Conclusion: The volume reduction rate gradually decreased, and 270 days were required for $50 \%$ volume reduction following decompression of OKC. The surgeon should be aware of this pattern to determine the timing for definitive enucleation.
\end{abstract}

Clinical relevance: The volume reduction rate and pattern of decompression of the OKC can be predicted and clinicians should be considered when treating OKC via decompression.

Keywords: Odontogenic keratocyst, Keratocystic odontogenic tumor, Decompression, Enucleation

\section{Background}

An odontogenic kearatocyst $(\mathrm{OKC})$ is a rare and benign but locally aggressive developmental cyst [1] and is also called as keratocystic odontogenic tumor. Under the microscope, OKCs vaguely resemble keratinized squamous epithelium. However, they lack rete ridges and often have an artefactual separation from their basement membrane. Treatment of OKC has stirred controversy because of the tumor's aggressive behavior and high recurrence rate.

The two common treatments of OKC at present are enucleation and decompression [2]. Enucleation followed by application of Carnoy's solution, liquid nitrogen cryotherapy, and radical surgery with resection is one of treatment of OKC [3]. However, these treatment methods may

\footnotetext{
* Correspondence: cancer7@yuhs.ac

${ }^{\dagger}$ Jin Hoo Park and Eun-Jung Kwak contributed equally to this work.

1 Department of Oral and Maxillofacial Surgery, College of Dentistry, Yonsei

University, 50-1 Yonsei-ro, Seodaemun-gu, Seoul 120-752, Korea

${ }^{2}$ Oral Science Research Institute, College of Dentistry, Yonsei University,

Seoul, Korea

Full list of author information is available at the end of the article
}

entail several complications. Alternative treatments are decompression which creates a cystic cavity which connects with the oral cavity [4] and is able cystic fluid to come out. After some decompression period, surgical enucleation is followed by decompression. If the lesion enlarges and approaches a vital structure, decompression is more recommended $[5,6]$.

This study was aimed to analyze the reducing pattern of decompression on mandibular OKC in terms of direction and speed of volume reduction and to determine the proper timing for secondary enucleation.

\section{Materials and methods}

This study conforms to the Declaration of Helsinki on medical protocol and ethics and was approved by the regional Ethical Review Board of Yonsei University Dental Hospital Institutional Review Board (IRB 2-2014-0031).

There were 90 subjects who were diagnosed with OKC from 2007 to 2013 at the Department of Oral and Maxillofacial Surgery, Yonsei University Dental Hospital. The exclusion criteria were other cystic lesions such as 
radicular cyst, dentigerous cyst, and unicystic ameloblastoma; an existing medical, physical, or mental condition that would impair the healing potential; syndromic craniofacial lesion such as basal cell nevus syndrome; or the absence of any of digitized pre-/post-operative CT and the site of lesion was limited only within mandible. Seventeen patients were included for this study base on the above inclusion and exclusion criteria.

Patients preliminarily diagnosed with OKC underwent an incisional biopsy of the cyst at the date of decompression. The patient's individual appliance for decompression tube was fabricated and located to the punctured area. During the follow-up period, self-irrigation through the decompression tube was applied. Surgeons checked reduction of cystic volume and bone formation via clinical and radiographic examination (panoramic view) every 2-3 months. When the cystic fluid exudate lessened clinically and radiography indicated the tumor had shrunk from important anatomical structures such as the inferior alveolar nerve, surgeons stopped decompression and began planning to enucleate the OKC (Fig. 1).

Measurement data such as volume and other metrics was collected by InVivo software (Anatomage, San Jose, Calif). Due to the intrinsic difficulty of applying automated image processing algorithms, heuristic approaches were widely applied in the data acquisition process. The size of the area was measured from indicated reference points along the cystic lesion in every axial cut of CT images. Volume was then approximated using Riemannian integration by summing up area values from each layer. The buccolingual (BL) and mesiodistal (MD) widths were measured in a similar manner from an image with the maximum area (Fig. 2). The three different investigators independently collected the data and analyzed it with the average values. The authors evaluated the effect of decompression of OKC in terms of volume change as well as MD and BL widths.

Estimation of coefficients $a$ and $b$ in the model was performed by standard regression in terms of square error (Fig. 3). Intrinsic assumption of normality of data was tested using Shapiro-Wilks test and other posterior verification methods, including QQ plot and residual test.

\section{Results}

The study group consisted of 17 patients, 8 males and 9 females, of average age 33.7 years (range 16-79 years). The average age of the 8 males was 29.8 years, and 9 females was 37.2 years. OKC prevalence was highest in the second and third decade (9/17 patients). The average initial volume was $34,020.85 \mathrm{~mm}^{3}$ and after decompression volume was $14,007.88 \mathrm{~mm}^{3}$. The average treatment period for all patients is 298 days of decompression (Table 1). The following formula presents the prediction of volume reduction according to elapsed time (Fig. 3). The volume of OKC undergoing decompression was reduced by 25,50 , and $75 \%$ over 103,270 , and 727 days (Fig. 3).

There was no significant directional indicator in the rate of reduction between buccolingual and mesiodistal widths (correlation coefficient 0.6626) (Fig. 4). The

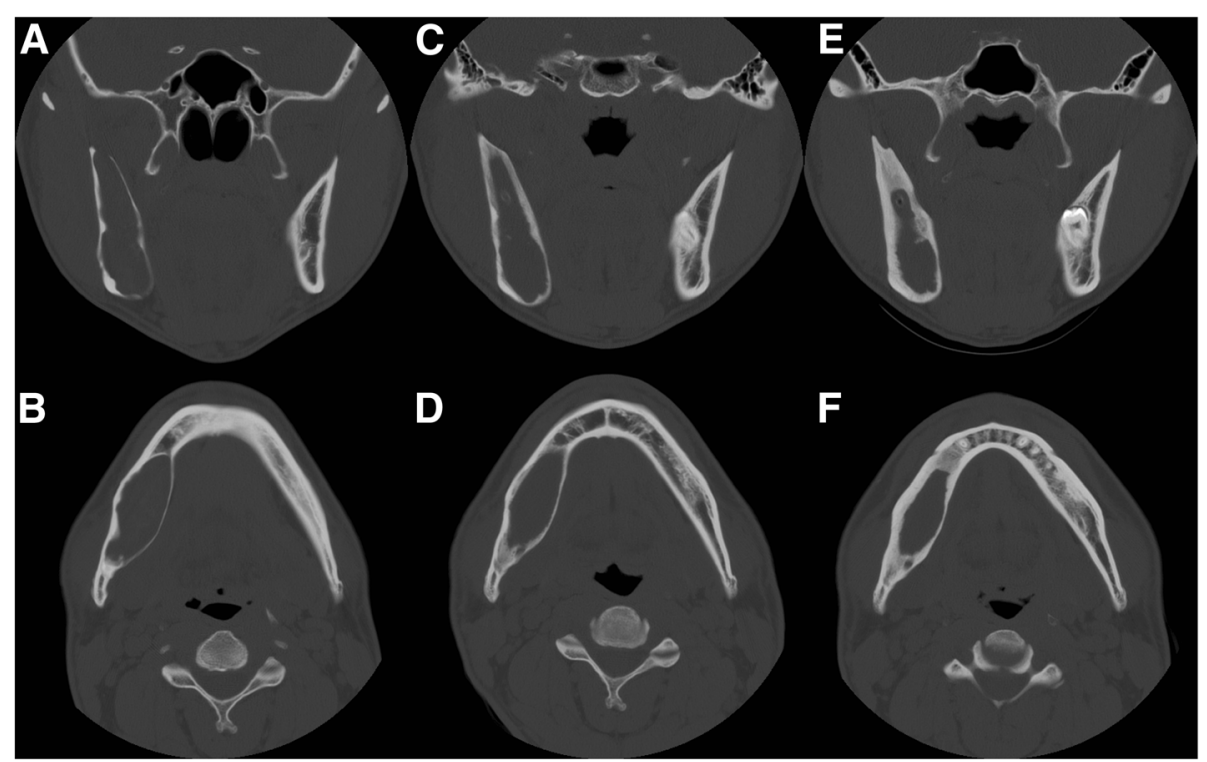

Fig. 1 Representive serial axial and coronal CT of OKC decompression. During the decompression for about 18 months, remarkable reduction in lesion size and increased cortical bone thickness with new bone formation is observed. (a, b initial, $\mathbf{c}, \mathbf{d} 6$ months decompression, e, $\mathbf{f}$ 18 months decompression) 


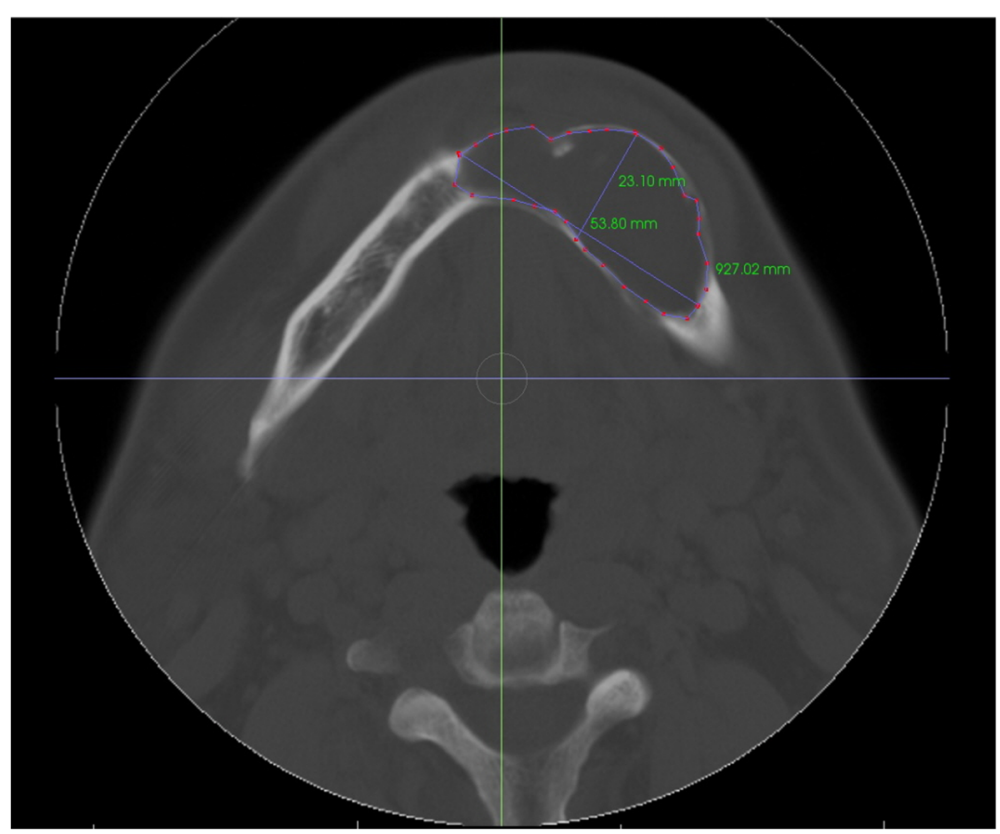

Fig. 2 The size of an area in axial cut and BL, MD width of lesion is measured

$V(t)=V_{\text {InITIAL }} \cdot \exp \left(a t+\frac{1}{2} b t^{2}\right)$

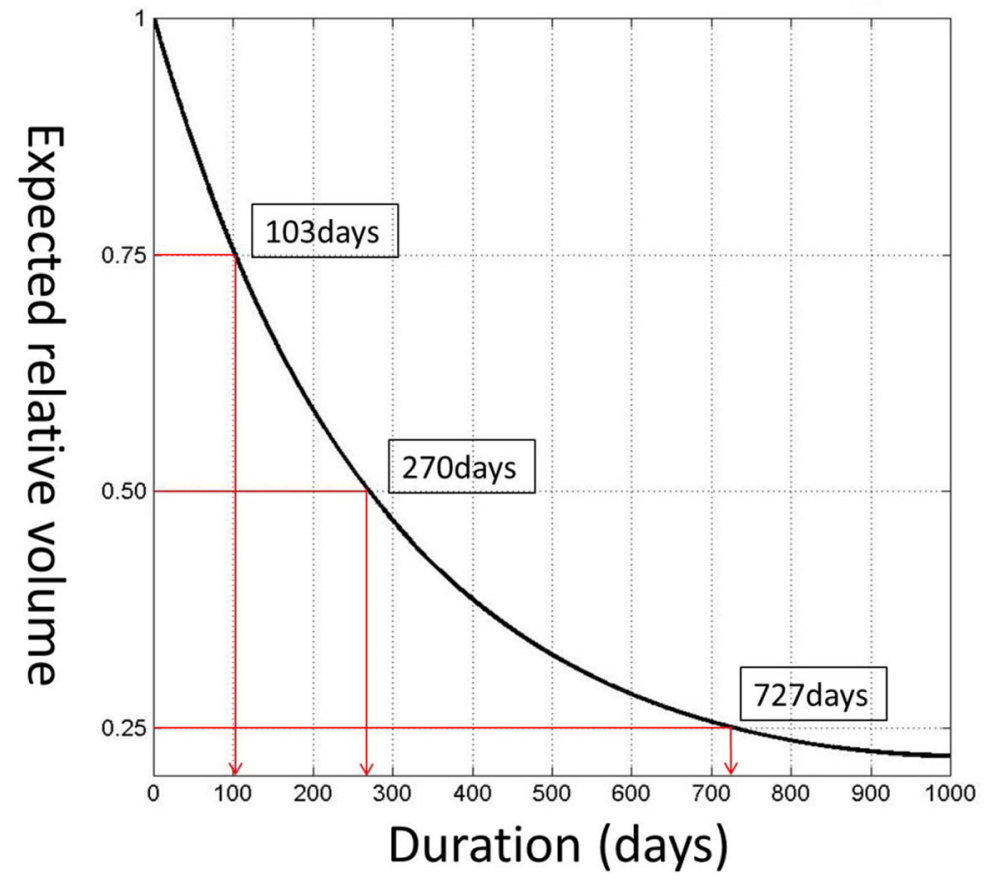

Fig. 3 The expected relative volume 
Table 1 Demographic of patients' information

\begin{tabular}{lll}
\hline Male: female & $1: 1.12$ & \\
Age & 33.72 & \pm 18.00 \\
Initial volume & $34,020.85 \mathrm{~mm}^{3}$ & $\pm 20,778.57$ \\
Volume reduction rate & $56.05 \%$ & \pm 16.83 \\
Duration of decompression & 298.35 days & \pm 183.02 \\
\hline
\end{tabular}

changes in volume as well as $\mathrm{MD}$ and BL diameters during the decompression period are shown (Fig. 5).

The average follow-up period is 776 days ( 2.1 years). Among 17 patients, the recurrence case is only 1 patient, observed 6.5 years after surgery.

\section{Discussion}

OKC is one of the most aggressive cyst among odontogenic cystic lesions. Though OKC is not known to metastasize, it can induce resorption, expansion of the jaw and change of the facial profile as it grows. Although in comparison with a small number of studies, prevalence in this study was higher in 10 to 30 -year-old patients, consistent with a previous study [7]. One-third of patients were asymptomatic and usually diagnosed by routine dental examination; $50 \%$ of them complained of jaw swelling and mild pain $[8,9]$. Though rare, conversion to malignant deviation such as primary intraosseous squamous cell carcinoma (PIOSCC) of the jaw is possible.

There are several studies on the differences in recurrence according to the treatment modalities of OKC. Kaczmarzyk et al. report that, the overall recurrence rate of OKC was $23.15 \%$. The recurrence rates were followed by treatment modalities were $0 \%$ for resection, $0 \%$ for enucleation with peripheral ostectomy and Carnoy's solution, $18.18 \%$ for enucleation with peripheral ostectomy, $26.09 \%$ for enucleation alone, $40 \%$ for marsupialization without following enucleation, and $50 \%$ for enucleation with Carnoy's solution [10]. The most obvious way to prevent recurrence of OKC is resection. But this is a very invasive surgical procedure. In a review study of OKC in Blanas et al., simple enucleation was reported to have a recurrence rate of $17 \%$ to $56 \%$ [11]. The recurrence rate of simple enucleation of OKC is very high. However there are several reports of a low recurrence rate after decompression following enucleation of OKC $(0-8.7 \%)[4,12-14]$.

The decompression and second-stage enucleation of OKC might be the first choice of treatment in terms of functional and esthetic reconstruction. In cases of huge cystic size and proximity with vital anatomic structures, decompression is the most proper treatment. During the decompression period, an opening by appliance allows for drainage of fluid which consists of OKC. This leads to decreased intracystic pressure [15] and bone formation along the periphery of the cystic wall, regardless of the direction of either MD or BL. Decompression and second-stage enucleation can thus be deemed beneficial in terms of decreased complications (nerve injury, pathologic fracture, discomfort).

The volume of OKC was reduced by 25,50 , and $75 \%$ over 103, 270, and 727 days of decompression. The surgeons in our department decided timing for enucleation based on the amount of discharging fluid and detachment from important anatomical structure in panoramic and CT images. The average decompression duration of study group was 298 days in this way. The time to enucleation based on the reduced exudate clinically and

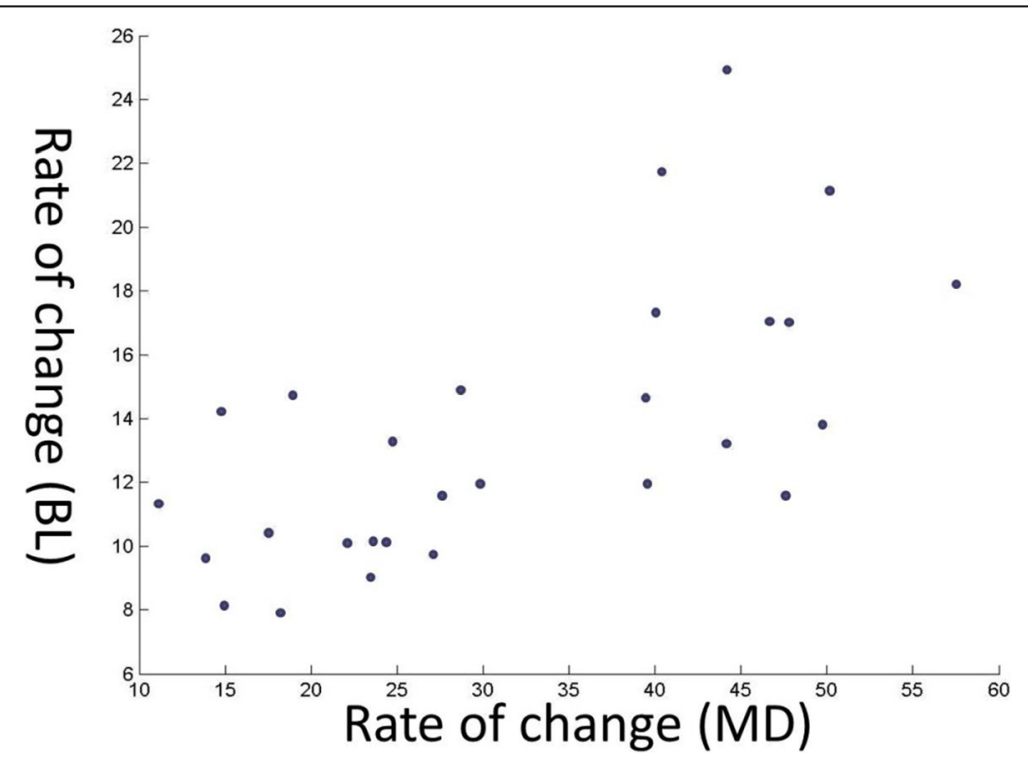

Fig. 4 Relation between $B L$ and $M D$ widths 


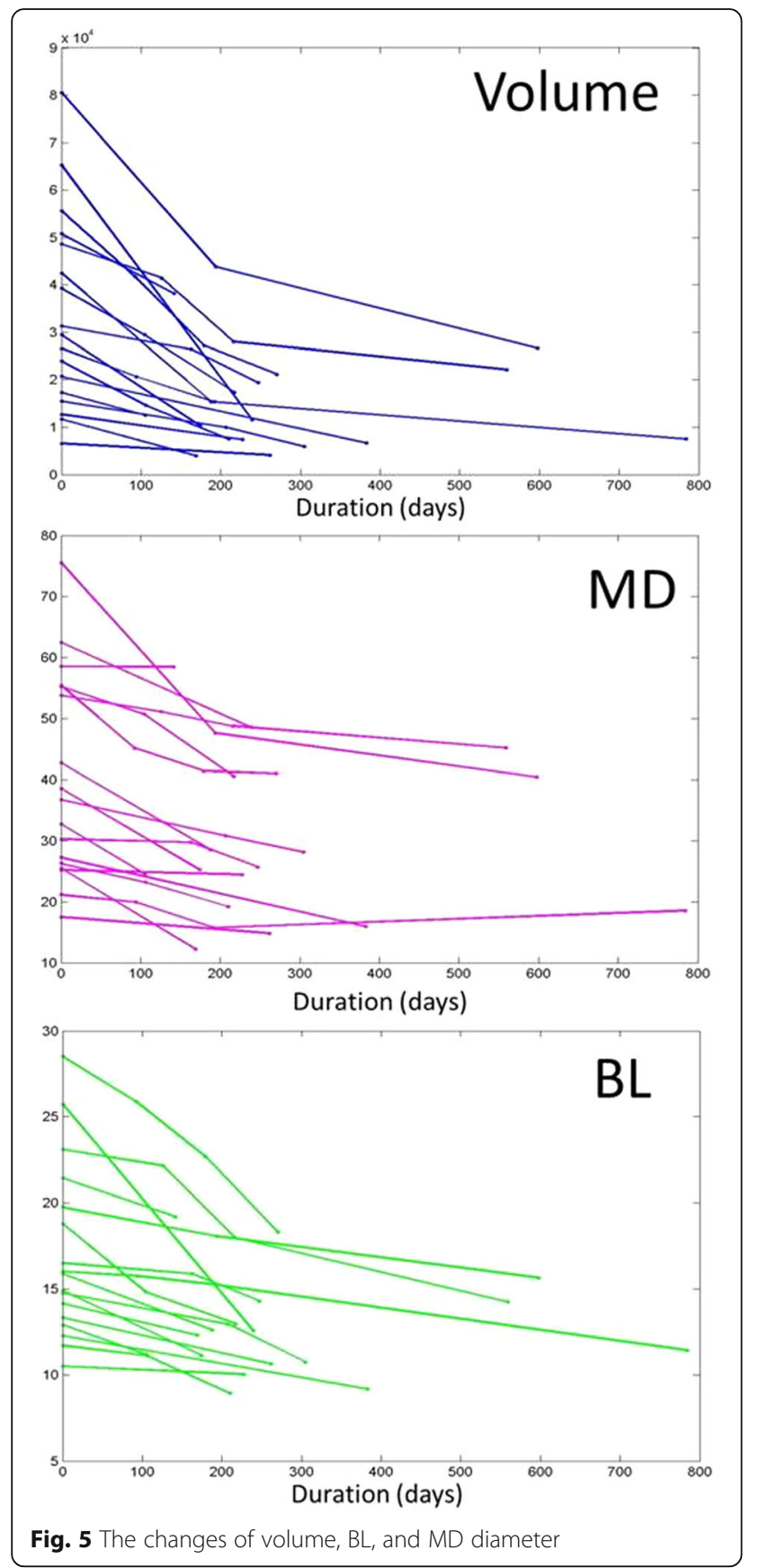

volume change as observed by the surgeon (298 days) was similar with the time of $50 \%$ reduction rate of OKC (270 days). Therefore, enucleation time set by the surgeon seems appropriate.

Tube length during decompression should be continuously adjusted as the OKC decreases in size. Amount of decrease can be confirmed by both panoramic view and CT. However, investigation by panoramic view is limited as evaluation of buccolingual dimension and volume are close to impossible [15]. In this respect, CT yields more information than 2D imagery. Therefore, panoramic images should be taken during periodic follow-up, CT being taken when there is a necessity for more accurate confirmation.

A relatively low recurrence rate was observed in this study when decompression was followed by enucleation. Moreover, the final pathology report was coincided with that of incisional biopsy performed in decompression procedure.

\section{Conclusion}

As a treatment of $\mathrm{OKC}$, decompression followed by enucleation was more conservative and effective than other procedures. Decompression preserves the important anatomical structures of the jaw and thus decreases complications such as functional and esthetic damage [16]. This study provides novelty in that it can predict the volume reduction of the decompressed OKC, which reduces in a half-life of 270 days. Although the rate of recurrence is low using this procedure, periodic checkups are necessary.

\section{Abbreviations}

BL: Buccolingual; CT: Computed tomography; KCOT: Keratocystic odontogenic tumor; MD: Mesiodistal; OKC: Odontogenic keratocyst; PIOSCC: Primary intraosseous squamous cell carcinoma

\section{Acknowledgements}

Not applicable.

\section{Funding}

This research was supported by Basic Science Research Program through the National Research Foundation of Korea (NRF) grant funded by the Ministry of Science, ICT \& Future Planning (NRF-2017R1C1B5075858).

\section{Availability of data and materials}

Data and material sharing applicable to this article

\section{Authors' contributions}

$J H P, E-J K, K S Y, Y-S J$, and H-DJ were responsible for the conception and design of the study/review/case series. JHP and E-JK were responsible for the acquisition of data, laboratory, or clinical/literature search. JHP, E-JK, and KSY were responsible for the analysis and interpretation of the data collected. $J H P, E-J K, Y-S J$, and H-DJ were responsible for the drafting of the article and/or the critical revision of the manuscript. H-DJ was responsible for the final approval and guarantor of the manuscript. All authors read and approved the final manuscript.

\section{Ethics approval and consent to participate}

This study conforms to the Declaration of Helsinki on medical protocol and ethics and was approved by the regional Ethical Review Board of Yonsei University Dental Hospital Institutional Review Board (IRB 2-2014-0031).

\section{Consent for publication}

Not applicable.

\section{Competing interests}

The authors declare that they have no competing interests.

\section{Publisher's Note}

Springer Nature remains neutral with regard to jurisdictional claims in published maps and institutional affiliations. 


\section{Author details}

'Department of Oral and Maxillofacial Surgery, College of Dentistry, Yonsei University, 50-1 Yonsei-ro, Seodaemun-gu, Seoul 120-752, Korea. ${ }^{2}$ Oral Science Research Institute, College of Dentistry, Yonsei University, Seoul, Korea. ${ }^{3}$ Department of Applied and Computational Mathematics and Statistics, University of Notre Dame, Indiana, USA.

Received: 18 November 2018 Accepted: 11 December 2018

Published online: 07 January 2019

\section{References}

1. Madras J, Lapointe H (2008) Keratocystic odontogenic tumour: reclassification of the odontogenic keratocyst from cyst to tumour. J Can Dent Assoc 74(2):165-165h

2. Kubota Y, Imajo I, Itonaga R, Takenoshita Y (2013) Effects of the patient's age and the size of the primary lesion on the speed of shrinkage after marsupialisation of keratocystic odontogenic tumours, dentigerous cysts, and radicular cysts. Br J Oral Maxillofac Surg 51(4):358-362

3. Stoelinga PJ (2001) Long-term follow-up on keratocysts treated according to a defined protocol. Int J Oral Maxillofac Surg 30(1):14-25

4. Anavi Y, Gal G, Miron H, Calderon S, Allon DM (2011) Decompression of odontogenic cystic lesions: clinical long-term study of 73 cases. Oral Surg Oral Med Oral Pathol Oral Radiol Endod 112(2):164-169

5. Nakamura N, Mitsuyasu T, Mitsuyasu Y, Taketomi T, Higuchi Y, Ohishi M (2002) Marsupialization for odontogenic keratocysts: long-term follow-up analysis of the effects and changes in growth characteristics. Oral Surg Oral Med Oral Pathol Oral Radiol Endod 94(5):543-553

6. Park SY, Shin YJ, Kim CH, Kim BJ (2015) Reconstruction of extensive jaw defects induced by keratocystic odontogenic tumor via patient-customized devices. Maxillofac Plast Reconstr Surg 37(1):37

7. de Molon RS, Verzola MH, Pires LC, Mascarenhas VI, da Silva RB, Cirelli JA et al (2015) Five years follow-up of a keratocyst odontogenic tumor treated by marsupialization and enucleation: a case report and literature review. Contemp Clin Dent 6(Suppl 1):S106-S110

8. Anand VK, Arrowood JP Jr, Krolls SO (1994) Malignant potential of the odontogenic keratocyst. Otolaryngol Head Neck Surg 111(1):124-129

9. Tan B, Yan TS, Shermin L, Teck KC, Yoke PC, Goh C et al (2013) Malignant transformation of keratocystic odontogenic tumor: two case reports. Am J Otolaryngol 34(4):357-361

10. Kaczmarzyk T, Mojsa I, Stypulkowska J (2012) A systematic review of the recurrence rate for keratocystic odontogenic tumour in relation to treatment modalities. Int J Oral Maxillofac Surg 41(6):756-767

11. Blanas N, Freund B, Schwartz M, Furst IM (2000) Systematic review of the treatment and prognosis of the odontogenic keratocyst. Oral Surg Oral Med Oral Pathol Oral Radiol Endod 90(5):553-558

12. August M, Faquin WC, Troulis MJ, Kaban LB (2003) Dedifferentiation of odontogenic keratocyst epithelium after cyst decompression. J Oral Maxillofac Surg 61(6):678-683 discussion 683-674

13. Brondum N, Jensen VJ (1991) Recurrence of keratocysts and decompression treatment. A long-term follow-up of forty-four cases. Oral Surg Oral Med Oral Pathol 72(3):265-269

14. Marker P, Brondum N, Clausen PP, Bastian HL (1996) Treatment of large odontogenic keratocysts by decompression and later cystectomy: a longterm follow-up and a histologic study of 23 cases. Oral Surg Oral Med Oral Pathol Oral Radiol Endod 82(2):122-131

15. Zhao Y, Liu B, Han QB, Wang SP, Wang YN (2011) Changes in bone density and cyst volume after marsupialization of mandibular odontogenic keratocysts (keratocystic odontogenic tumors). J Oral Maxillofac Surg 69(5):1361-1366

16. Morais de Melo W, Pereira-Santos D, Sonoda CK, Hochuli-Vieira E (2012) Decompression for management of keratocystic odontogenic tumor in the mandible. J Craniofac Surg 23(6):e639-e640

\section{Submit your manuscript to a SpringerOpen ${ }^{\circ}$ journal and benefit from:}

- Convenient online submission

- Rigorous peer review

- Open access: articles freely available online

- High visibility within the field

- Retaining the copyright to your article

Submit your next manuscript at $\boldsymbol{\nabla}$ springeropen.com 